\section{Bringing Science to Clinical Practice}

Life is complicated. In my practice, I care for patients with pancreatic adenocarcinoma, and it's not easy. Available treatments, although improving, are still sparse. Often these patients don't live very long. So it's not surprising that they are keenly interested in having their tumors tested for things that might increase their therapeutic options, such as genetic mutations or amplifications or protein expression. And we doctors want to provide those options. So we faithfully send biopsies out for testing and hope that the findings will steer us toward a clinical trial when the time is right. Often, however, no trial is available, and we're faced with the dilemma of having an actionable finding with no body of evidence to support use of the drug in the context of the disease at hand.

Right now, I have a patient-a young woman with pancreatic cancer-who, surprisingly, has a somatic BRCA mutation and HER2 amplification. At first, I found it reassuring that we might have some different options to pursue. But when her disease began to progress after standard therapy, we couldn't find an open trial with a poly(ADP)-ribose polymerase (PARP) inhibitor or with an antibody or small molecule targeting HER2. Her disease was progressing rapidly, and we made a decision to start trastuzumab and pertuzumab, off-label. I acknowledge having no evidence on which to base this decision. Long ago, a negative trial evaluating trastuzumab was performed in HER-positive pancreatic cancer. Of course, the effort preceded our current techniques for measuring HER2 positivity and we didn't appreciate the concomitant advantage of blocking HER2 dimerization.

Although my patient was prepared to pay for the off-label treatment out-ofpocket, a miracle happened and her insurance authorized the treatment. It's too soon to know whether this strategy will help her, and I lie awake each night wondering if we did the right thing.

As long as I can remember, off-label use of drugs has been common in oncology. We don't have a method for tracking the outcomes of off-label use, but our formerly lenient payer system accommodated the practice. However, with the cost of cancer care soaring - largely because of expensive new drugs - the landscape is changing and we must find a way to use these drugs conservatively. NCCN is tackling this head on. In this issue, you will find an NCCN Working Group Report, "Designing Clinical Trials in the Era of Multiple Biomarkers and Targeted Therapies." This represents the beginning of an effort within NCCN to create a framework for establishing the value of targeted therapy in unique subsets. We already know that, in common diseases, even a small fraction of patients defined by a common mutation can serve as a sizable cohort for clinical trials. But how do we handle unique patients or really small subsets? How do we create an evidence base when large randomized clinical trials are not feasible?

I like this working group report for its educational value too. If you want a quick refresher on the rationale for and the use of new targeted therapeutics, this is for you. And in terms of providing direction on unique patients or small subsets, it's just a start. In a few months, you'll notice a new feature in JNCCN, "Molecular Insights in Patient Care." This feature will explore the use of targeted therapeutics in unique patients, and we will be asking contributors to lay out the approach needed to extend their observations to broader clinical application.

I really hope my patient gets better. Either way, I hope the outcome can be catalogued and that everyone can learn from our experience as I hope to learn from others. It's just a beginning. But you have to start somewhere.

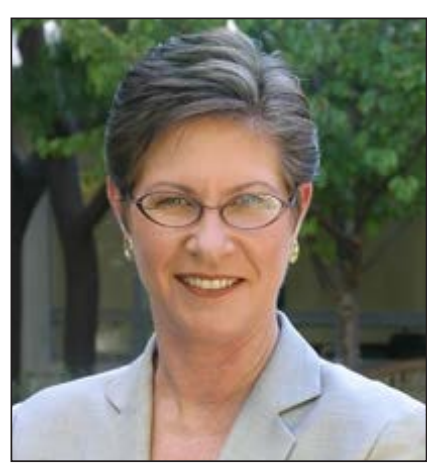

Margaret Tempero, MD

Dr. Tempero is a Professor of Medicine and Director of the UCSF Pancreas Center, and the editor-in-chief of JNCCN. Her research career has focused on pancreatic ductal adenocarcinoma especially in the area of investigational therapeutics. Dr. Tempero has served on the ASCO Board of Directors and as ASCO President. She co-directed the AACR/ASCO Methods in Clinical Cancer Research and taught this course and similar courses in Europe and Australia. She was founding Chair of the $\mathrm{NCl}$ Clinical Oncology Study Section (CONC) and served as a member and chair of the $\mathrm{NCl}$ Board of Scientific Counselors Subcommittee A. She is on the External Advisory Boards of the Pancreas SPOREs at Mayo Clinic and at $\mathrm{UAB} /$ Minnesota and the GI SPORE at the University of Arizona. She is, or has been, on the Scientific Advisory Boards of the Lustgarten Foundation, the Pancreatic Cancer Action Network, the V Foundation, The Alberta Canada Cancer Board, and the EORTC. She served as a member of the Oncology Drug Advisory Committee for the FDA.

She has served as Deputy Director and Interim Director for the UNMC Eppley Cancer Center. She is Chief Emeritus of the Division of Medical Oncology at UCSF and served as Deputy Director and Director of Research Programs at the UCSF Helen Diller Family Comprehensive Cancer Center.

The ideas and viewpoints expressed in this editorial are those of the author and do not necessarily represent any policy, position, or program of NCCN. 